\title{
Update on the efficacy, safety, and adherence to treatment of full length parathyroid hormone, PTH (I-84), in the treatment of postmenopausal
} \section{osteoporosis}

\author{
This article was published in the following Dove Press journal: \\ International Journal of Women's Health \\ 17 November 2009 \\ Number of times this article has been viewed
}

\author{
Luca Pietrogrande \\ Dipartimento di Medicina Chirurgia \\ e Odontoiatria Polo San Paolo, \\ Università degli Studi di Milano, \\ Milan, Italy
}

Correspondence: Luca Pietrogrande U.O. Ortopedia e Traumatologia A.O. San Paolo, via di Rudinì, 8, 20I42 Milano, Italy

Tel +390250323107

Fax +390250323111

Email luca.pietrogrande@unimi.it

\begin{abstract}
Full length (1-84) parathyroid hormone (PTH) was introduced in Europe as a treatment for postmenopausal osteoporosis in 2006. The efficacy of PTH (1-84) in the prevention of vertebral fractures is very high, and is similar to that of teriparatide. Its action in the prevention of femoral fractures has yet to be fully demonstrated, but the incidence of such fractures in trials was very low, and a decrease in nonvertebral fractures was seen in high-risk patients. The effect on bone mineral density (BMD) was clearly demonstrated in the spine and also in the hip. The effects on BMD were evident and increased progressively with treatment until 36 months. After its discontinuation there was a clear decrease in BMD if no antiresorptive treatment was initiated. Increases in bone volumetric density and bone volume in trabecular sites were also reported. Moreover, a bone volume increase was detected in cortical sites. Hypercalcemia and hypercalciuria are frequent consequences of PTH treatment, but rarely have clinical effects and are usually well controlled by reducing calcium and vitamin D supplementation.
\end{abstract}

Keywords: PTH (1-84), full-length parathyroid hormone, osteoporosis treatment

\section{Introduction}

The anabolic effect of parathyroid hormone (PTH) in cancellous bone was suspected in primary hyperparathyroidism as long as 20 years ago. ${ }^{1-3}$ Its use as an anabolic agent in the treatment of osteoporosis is based on abundant human and animal literature, which led to its intermittent low-dose administration. ${ }^{4-8}$ The exact mechanisms of PTH's double effect (anabolic with intermittent low dose, and catabolic with continuous high dose) are still not completely clear, even though it is well understood that PTH acts directly through PTHR1 receptors on osteoblasts to stimulate bone formation and also indirectly stimulates differentiation and development of osteoclast cells, leading to active bone resorption by increasing the receptor activator of nuclear factor- $\mathrm{\kappa B}$ ligand and its decoy receptor osteoprotegerin (RANKL/OPG) gene expression ratio., ${ }^{9,10}$ The anabolic effect is prevalent during intermittent administration, while osteoclast activation occurs with continuous exposure (eg, in primary hyperparathyroidism) ${ }^{11-14}$ The anabolic action is observed early in the treatment, while the catabolic action appears later. ${ }^{15}$

The use of PTH as an anabolic agent in the treatment of postmenopausal osteoporosis is relatively recent and at present only two different forms of PTH are licensed for this purpose: PTH (1-84), or full length PTH, and PTH (1-34), or teriparatide. In the US only teriparatide is approved for treatment of postmenopausal osteoporosis, while in Europe 
both are available for this use. Teriparatide has also been licensed for glucocorticoid-induced and male osteoporosis. Other forms of truncated PTH, PTH (1-31), PTH (1-36), and PTH (1-38), have been studied as treatments for osteoporosis, but no phase III trials have been completed. ${ }^{16}$

The commercially available PTH (1-84) (Preotact $^{\circledR}$; Nycomed, Denmark) is manufactured using a strain of Escherichia coli modified by recombinant DNA technology. The medicinal product is supplied in a dual-chamber cartridge: one chamber contains the PTH in powder, the other a sterile solvent for reconstitution. The cartridge is inserted into an injection pen, which injects subcutaneously a dose of $71.4 \mu \mathrm{L}$ containing $100 \mu \mathrm{g}$ PTH. Each cartridge contains 14 doses and, after reconstitution, has to be stored in a refrigerator $\left(2\right.$ to $\left.8{ }^{\circ} \mathrm{C}\right) .{ }^{17}$

This review analyses the most recent literature on the clinical use of PTH (1-84), focusing on data on efficacy, safety, and adherence to treatment.

\section{Clinical trials involving PTH (I-84)}

Two large clinical studies - the Treatment of Osteoporosis with PTH (TOP) study, ${ }^{18}$ with its Open Label Extension Study (OLES), ${ }^{19}$ and the PTH and alendronate for osteoporosis $(\mathrm{PaTH})$ clinical trial ${ }^{20,21}$ - laid the foundations for the use of PTH (1-84) in the treatment of postmenopausal osteoporosis and provided most of the data on its efficacy and safety. A number of other studies, some still only at a preliminary stage, offer useful information on the clinical use of PTH (1-84). These include the phase II safety and efficacy trial, ${ }^{4}$ the Prevention of Osteoporosis in Women on Estrogen Replacement (POWER) study, ${ }^{22}$ the Treatment Extension Study (TRES), ${ }^{23}$ and the Preotact ${ }^{\circledR}$ after a break (PEAK) study. ${ }^{24}$ Other data are taken from abstracts presented at recent international osteoporosis meetings. The features of the most important studies will be outlined below.

\section{Phase II safety and efficacy trial ${ }^{4}$}

This is a 12-month, double-blind, placebo-controlled, dosefinding study, which investigated the efficacy and safety of human PTH (1-84) in the treatment of postmenopausal osteoporosis. It enrolled 217 50- to 75-year-old North American postmenopausal women with a lumbar spine bone mineral density (BMD), measured by double X-ray absorptiometry (DXA), <-2.5 standard deviations below the mean peak bone mass in young women (T-score ). They had no significant medical problems that might affect skeletal homeostasis or vertebral abnormalities, and had not received treatment for osteoporosis recently. The patients were randomized to receive PTH (1-84) at different dosage (50, 75, and $100 \mu \mathrm{g})$ and placebo, and all patients received daily supplementation of calcium carbonate (500 to $1000 \mathrm{mg}$ ) and vitamin D (400 IU). The primary endpoint was to assess the percentage change in vertebral BMD from baseline. BMD and bone mineral content (BMC) at the femoral site, as well as bone marker values were also measured, adverse events collected, and compliance assessed.

\section{The TOP study with OLES and TRES extensions}

The TOP ${ }^{18}$ is an 18-month, randomized, double-blind, placebo-controlled, parallel-group study performed in 168 centers in 9 countries, enrolling 2532 postmenopausal women with low BMD at the lumbar spine. Women 45 to 54 years of age $(8.1 \%)$ were included if BMD was $\leq-3.0$ at the lumbar spine, femoral neck or total hip, or if BMD was $\leq-2.5$ and they had 1 to 4 prevalent vertebral fractures. Women 55 years of age or older $(11.4 \%>75$ years $)$ were included if BMD was $\leq-2.5$, or if BMD was $\leq-2.0$ and they had 1 to 4 fractures. Women were also enrolled even if mild hypercalcemia or hypercalciuria (ie, serum calcium 2.55 to $2.66 \mathrm{mmol} / \mathrm{L}$ [10.2 to $10.7 \mathrm{mg} / \mathrm{dL}$ ] and 24-hour urine calcium $\geq 7.6 \mathrm{mmol}$ [302 $\mathrm{mg}$ ]) were present. History of metabolic bone disease, significant renal or hepatic disorders and recent osteoporosis treatment were exclusion criteria. Of the enrolled women 1286 received $100 \mu \mathrm{g}$ PTH (1-84) and 1246 placebo, and all received calcium citrate $(700 \mathrm{mg} /$ day $)$ and vitamin D (400 U/day). The primary endpoint was the occurrence of new or worsened vertebral fractures identified by radiography. Secondary end outcomes included changes in BMD (at the spine, hip, distal radius, and total body), in bone turnover markers, and in quantitative computed tomography (QCT) for 122 women only. Nonvertebral clinical fractures and bone loss were reported as adverse events. The study was completed by 1701 women $-877(70 \%)$ in the placebo group and 824 (64\%) in the PTH group. The drop-out rate was high in both groups (30\% and $26 \%$ in the PTH and placebo group respectively) but a specific cause has not been identified. Even if more patients in the PTH group withdrew consent because of adverse events (16\%), only a small proportion of patients who experienced adverse events discontinued participation.

From the TOP study, 1681 patients continued into OLES to assess the safety and efficacy of prolonged use of PTH. From the PTH group, 781 patients continued the same treatment for a further 6 months (a total of 24 months) and were monitored for 12 months after discontinuation; from the placebo 
group 900 patients received the same treatment as the TOP PTH group for a further 18 months. Changes in BMD, bone turnover markers, and adverse events were monitored. ${ }^{19}$

One hundred three women, who had been treated for 18 months in OLES, were screened and 91 included in the intention to treat (ITT) group in TRES, after an approximate 2-month break in PTH treatment between OLES and TRES. These patients were treated with PTH (as in the TOP study) for a total of 36 months. Changes in BMD and bone turnover markers and adverse events were monitored. ${ }^{23}$

Figure 1 shows the flow chart of all three studies.

\section{The PaTH study ${ }^{20,21}$}

The PaTH study is a randomized, double-blind clinical $1+1$ year trial to evaluate whether the concurrent administration of PTH (1-84) and alendronate would increase bone density more than the use of either one alone, in the first year, and whether antiresorptive therapy is required to maintain BMD increase after 1 year of PTH (1-84) treatment (100 $\mu \mathrm{g} /$ day). A total of 238 postmenopausal women (not using bisphosphonates) with low BMD at the hip or spine ( $\mathrm{T}$ score $<-2.5$, or a $\mathrm{T}$ score $<-2.0$ with an additional risk factor for osteoporosis) were randomly assigned to one of four different regimens - daily treatment with PTH in the first and placebo in the second year, PTH in the first and alendronate in the second year, $\mathrm{PTH}+$ alendronate in the first, or alendronate for two years. All patients received a supplementation with calcium carbonate $(500 \mathrm{mg})$ and vitamin D (400 IU) (Figure 2).

BMD changes at the lumbar spine, hip, and distal radius were primary endpoints, assessed by DXA. In a subset of 204 patients, QCT was also performed at the spine and the hip. Markers of bone turnover were measured, adherence and adverse events were assessed.

\section{The POWER study 22}

The POWER study is a randomized, double-blind, placebo-controlled, parallel-group study conducted at

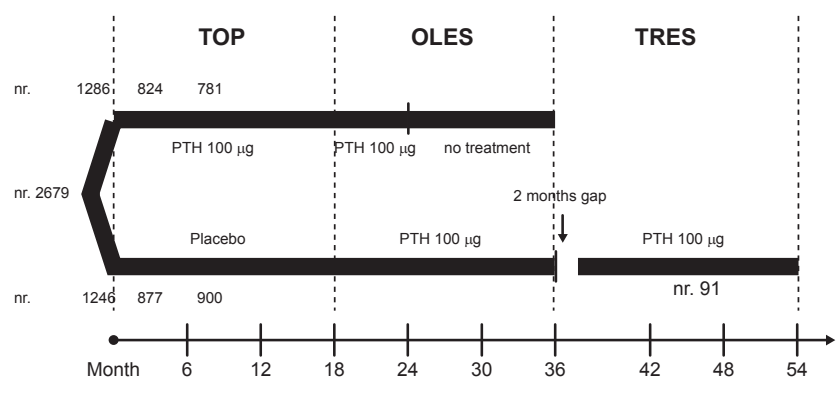

Figure I The flow of the TOP, OLES and TRES studies.
25 clinical centers, designed to evaluate the effects of adding PTH (1-84) treatment to women receiving stable hormone replacement therapy (HT). Initially planned for 24 months, it was modified, following European Medicines Agency recommendations on estrogens in osteoporosis, and the treatment stopped at 18 months. Consequently, '18-month' data represent evaluations conducted between 12 and 18 months and '24-month' data represent measurements between 18 and 24 months.

The study recruited a total of 187 women, 45 years old or more, a minimum of 1 year after menopause, on stable HT, and with a BMD T-score $<-2.0$. Only 124 completed the 18 months of treatment, 69 on HT (HT group) and 55 on HT and PTH (HT + PTH group), all with calcium (700 mg/day, the salt is not specified) and vitamin D (400 U/day) supplementation. The primary efficacy endpoint was change in lumbar spine BMD; secondary endpoints were change in hip and distal radius BMD, bone turnover markers, height, and incidence of vertebral and clinical fractures.

\section{The PEAK study ${ }^{24}$}

The ongoing PEAK study is a 3-year, randomized, multicenter, open-label, phase IIIb trial investigating lumbar spine BMD changes in postmenopausal women with primary osteoporosis. In the first year of the trial all patients were treated with PTH (1-84), and in the second year

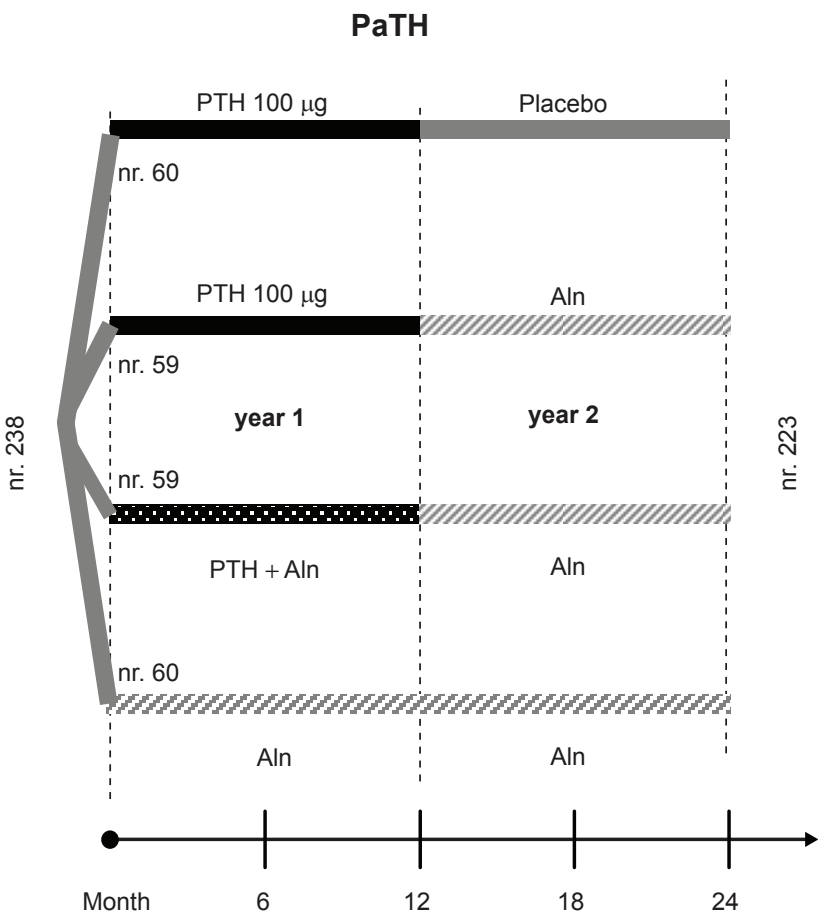

Figure 2 The 4 branches of the 2 year PaTH study. 
all patients are being treated with risedronate. In the third trial year patients will be randomized and divided equally to either continue risedronate treatment or receive PTH (1-84). Details on calcium and vitamin D supplementation are not given. The primary endpoint is to verify increase in lumbar spine BMD, but the effect of PTH on calcium homeostasis is also being investigated. Postmenopausal women (408) aged $>50$ years with primary osteoporosis (lumbar spine T-score $\leq-3.0 \mathrm{SD}$ ) were enrolled even if pre-treated with bisphosphonates. Women with a serum calcium $>2.55 \mathrm{mmol} / \mathrm{L}$ at baseline and/or calcium metabolism abnormalities were excluded.

Only interim analyses are available - one assessing calcium values during the first year of PTH, another evaluating BMD changes in subjects (27) treated with risedronate or alendronate for more than 6 months in the previous year.

\section{Efficacy}

The efficacy of PTH (1-84) in the treatment of osteoporosis has been evaluated with various endpoints, of which the most important is the incidence of new fractures, followed by increases in densitometric measurements (using DXA (Figure 3) and QCT scans) and variations in bone turnover parameters.

\section{Prevention of fractures}

The efficacy of PTH (1-84) in the prevention of new vertebral fractures in women with or without a prevalent fracture at baseline was demonstrated in the TOP study. In this trial the incidence of new or worsened vertebral fractures was significantly $(P=0.001)$ lower in treated patients $(1.4 \%)$ than in the placebo group (3.4\%), with a $58 \%$ (95\% confidence interval [CI] 0.24 to 0.72 ) reduction in relative risk (RR). The RR reduction for a first vertebral fracture was $68 \%$ (95\% CI 0.14 to 0.75$)(P=0.006)$ in patients without a prevalent fracture at baseline. The number of patients that needed to be treated (NNT) for a median of 18 months to prevent a new or worsened vertebral fracture was 51 (32 to 129). The NNT was 71 (42 to 422) in patients without a baseline fracture, and 22 (11 to 422 ) in those with a baseline fracture. ${ }^{18}$ Recently, in a reanalysis of the TOP trial data, the efficacy of PTH in the prevention of a new fracture was demonstrated to be greater in patients with a low lumbar BMD (T-score $<-3.0$ ) at baseline, with a $74 \%$ RR reduction $(1.3 \%$ vs $5.1 \% ; P<0.001)$, or in patients older than 60 years, with a $64 \%$ RR reduction $(1.5 \%$ vs $4.2 \% ; P<0.001){ }^{25}$

During the OLES few new vertebral fractures were seen $-1(1.7 \%)$ in the group treated with PTH (1-84) for a further 6 months, $2(1.9 \%)$ in the 12-month placebo-treatment

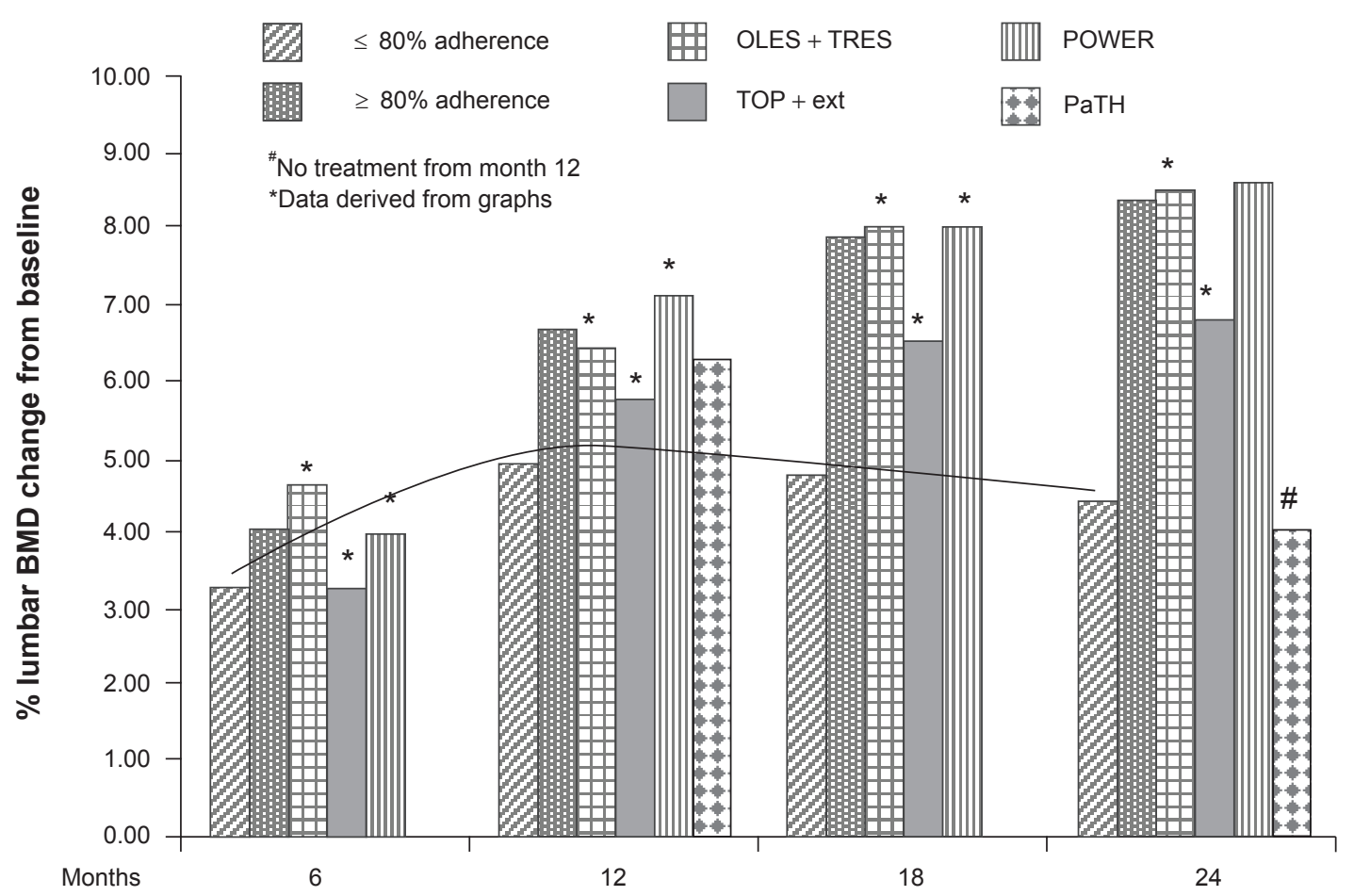

Figure 3 Comparison of percentage changes in lumbar spine BMD in patients treated with PTH (I-84) in the TOP, OLES, POWER and PaTH studies, and reanalysis of data for highly compliant patients. The line shows that the trend of noncompliant patients of all the other studies. A different trend is also seen for patients that stopped PTH treatment after 12 in the PaTH study. 
group, and 4 worsened vertebral fractures in the former PTH (1-84)-treated group. The authors suggest that the low number of new fractures, during extended PTH treatment and after its cessation, confirms the effect of PTH in the prevention of osteoporotic fractures. ${ }^{19}$

In the TOP study there was no detectable effect of PTH on nonvertebral fractures $(5.8 \%$ and $5.6 \%$ in the placebo and PTH groups, respectively). This may be because the population enrolled was younger and with less severe disease than in the studies that showed reduced nonvertebral fractures in patients treated with teriparatide. ${ }^{26}$ However, a post-hoc analysis of a high-risk subgroup of 231 patients with lumbar spine BMD T-scores $<-3$ showed a $42.8 \%$ statistically nonsignificant reduction in nonvertebral fractures. ${ }^{27}$

There were insufficient numbers of vertebral and clinical fractures to permit statistical analysis in the POWER trial. Three subjects in each treatment group experienced a clinical fracture during the study, only one of these in the spine in the HT-alone group. ${ }^{22}$

In the PaTH study, after 2 years, 21 women (8.8\%) had 1 or more clinical fractures, but no differences among groups were detected. ${ }^{21}$

\section{Areal densitometry}

The efficacy of PTH has been documented through changes in BMD at various sites in many studies. In the TOP trial, at month $18 \mathrm{PTH}$ treatment increased lumbar spine BMD by $6.5 \%$, compared with a $0.3 \%$ decrease in the placebo group $(P=0.001) .{ }^{18}$ There were also significantly greater increases in the PTH group in total hip $(2.1 \%)$, femoral neck $(2.5 \%)$, and trochanter BMD $(1.6 \%) .{ }^{28}$ At the distal radius BMD decreased in the $\mathrm{PTH}$ group by a mean of $3.4 \%$ $(P=0.001) .^{18}$

Treatment extension for up to 24 months in OLES resulted in a continued increase in BMD from baseline, $6.8 \%$ in lumbar spine and $2.2 \%$ in femoral neck. ${ }^{17}$

The patients treated in OLES and TRES for 36 months showed a progressive increase in lumbar spine and total hip BMD for the first 18 months ( $8.0 \%$ and $2.1 \%$ respectively). There was a small decrease (to $7.0 \%$ and $1.7 \%$ ) during the approximate 2-month break without treatment. Lumbar spine BMD further increased until month 24 of PTH treatment $(8.5 \%)$ remaining stable for the final 12 months. Total hip BMD increased progressively above OLES baseline by $3.4 \%$ at month $36 .^{23}$

The 24-month data from the TOP and OLES studies was analyzed recently by dividing the population into subjects with high adherence $(\geq 80 \%)$ and low adherence $(<80 \%)$ to the therapy. It was found that the BMD increased by $8.38 \%$ and $4.46 \%$ in lumbar spine, and by $2.58 \%$ and $1.53 \%$ in femoral neck, in the high- and low-adherence groups respectively. ${ }^{29}$

Also in the PaTH study, after 1 year of treatment with PTH (1-84), the increase in BMD was substantial in lumbar spine $(6.3 \%)$, and very small in total hip $(0.3 \%)$. A reduction $(-3.4 \%)$ in BMD was seen at the distal radius. In the PTH + alendronate and alendronate-alone groups there were lesser increases in lumbar spine BMD (4.6 and 6.1\%, respectively), but greater increases at the total hip and lesser decreases at the distal radius $\left(-1.1 \%\right.$ in the combination therapy group) ${ }^{20}$

After the second year of PaTH the BMD increased in all four groups at the lumbar spine. The maximum increase at the lumbar spine, total hip, and femoral neck $(12 \%, 4 \%$, and $4 \%$, respectively) was in the group treated with PTH in year one and with alendronate in year two. The minimum increase was in the group treated the first year with PTH and the second with placebo $(4 \%, 0 \%$, and $1 \%)$. The same intermediate increase $(8 \%, 3 \%$, and $3 \%)$ was also seen in the other two groups. ${ }^{21}$

In the POWER study increases in spine and femoral BMD were detected in both groups, with a significantly greater increase in the HT + PTH group at the lumbar spine at month 24 (difference $6.5 \%, P=0.001$ ) and at the femoral neck at month 18 (difference $1.8 \%, P=0.024$ ). A decrease in BMD in PTH-treated women was detected at the distal radius, but the difference was not significant for the HT-alone group. ${ }^{22}$

The interim analysis of the PEAK trial showed a median increase in lumbar spine BMD of $4.3 \%$ in 27 patients pre-treated with a bisphosphonate. The upper confidence limit was higher than $3 \%$, and therefore pre-treatment with a bisphosphonate does not seem to reduce the likelihood of a gain in BMD with PTH (1-84) therapy. ${ }^{24}$

\section{Volumetric densitometry}

In the TOP trial increases were detected at month 18 in trabecular BMD at the spine $(37.6 \%, P=0.001)$, total hip (4.7\%, $P=0.001)$, femoral neck $(4.5 \%, P=0.001)$, and trochanter $(4.3 \%, P=0.001)$, but there were also increases in total hip cortical volume $(9.0 \%, P=0.003)$ and total hip cortical volumetric bone content $(4.8 \%, P=0.040)$. A decrease was seen in the total hip cortical volumetric BMD $(-4.7 \%, P=0.001) .{ }^{18}$

In the PaTH study the volumetric BMD of trabecular bone increased at both the spine and the hip in all four groups during the two years. After the first year the increase in volumetric density of trabecular bone at the spine was 
$25.5 \%$ in the PTH group, $12.9 \%$ in the PTH + alendronate group, and $10.5 \%$ in the alendronate group. At month 24 in the PTH + alendronate group the increase was higher compared with other treatment regimens, 31\% at the spine ( $P=0.001$ vs baseline and other groups) and 13\% at the hip $(P=0.001$ vs baseline). Also the patients treated with combination therapy and alendronate showed a significant increase ( $11 \%, P=0.001$ vs baseline) in hip trabecular BMD. ${ }^{21}$ The volumetric BMD in cortical bone at the hip decreased in all groups (from $-1 \%$ to $-3 \%$ ) - the changes were significant except in the combination therapy and alendronate group. Significant cortical hip volume increases were detected in all groups, $1 \%$ in PTH/placebo, 6\% in PTH + alendronate and continued alendronate, and $7 \%$ in combination therapy/ alendronate groups. ${ }^{21}$

An interesting analysis of the data from the PaTH study was conducted to correlate structural indices assessed by hip structural analysis (HSA) ${ }^{30}$ and volumetric QCT. ${ }^{44}$ The DXA-derived HSA and QCT-derived indices of structural geometry in postmenopausal women who received 1 year of PTH followed by 1 year of alendronate were significantly associated with each other at baseline and showed similar improvements at 24 months. ${ }^{31} \mathrm{~A}$ finite element analysis with simulation for a sideways fall was performed on the hip QCT scans of 162 of these subjects. The changes in strength from baseline were statistically significant for all four groups at year one, but only for three groups at year two - the change seen in the PTH-placebo group was, in fact, only $1.17 \%$ from baseline. Changes in the trabecular density were the major cause of strength increases, regardless of treatment group, but changes also in cortical density and mass played a role, the degree of which depended on treatment. ${ }^{32}$

\section{Bone turnover}

The use of bone turnover markers as indices of efficacy is based upon the concept of the 'anabolic window', ie, the activation latency between formation and resorption markers. ${ }^{33}$

Bone formation markers rapidly increased from baseline in PTH-treated patients in all studies. Bone alkaline phosphatase (bALP) increased in the TOP study, approximately $30 \%$ at month $1,100 \%$ at months 6 and 12 , and $80 \%$ at month 18 in the PTH group, ${ }^{18}$ and in the POWER study, $98 \%$ at month 12 and $115 \%$ at month 24 in the PTH + HT group. ${ }^{22}$ The elevation of bALP decreased slightly in the OLES extension of the TOP study, remaining 27\% above the TOP baseline. ${ }^{29}$

Osteocalcin significantly increased dose-dependently in the PTH treated group in the phase II study - approximate percentage changes are derived from a graph and they range from $20 \%$ with $50 \mu \mathrm{g}$ to $45 \%$ with $100 \mu \mathrm{g}$ at month 1 , from $60 \%$ to $120 \%$ at month 6 , and from $75 \%$ to $150 \%$ at month $12 .{ }^{4} \mathrm{~N}$-propeptide of type 1 collagen (P1NP) increased after 1, 3, and 12 months of PTH therapy (changes derived from a graph were approximately $90 \%, 140 \%$, and 150\% respectively) in the PaTH study, and rapidly decreased after treatment suspension. ${ }^{21}$

Bone resorption markers also increased in all studies, but this increase was somewhat delayed in comparison with the change in bone formation markers in the TOP and PaTH studies. In the TOP study, a significant increase in urinary $\mathrm{N}$-telopeptides of type 1 collagen (NTx) was detectable only from month 6 (approximately 150\%) to month $18(85 \%){ }^{4}$ Women in the PTH-alone group in the PaTH study showed an increase in serum C-terminal telopeptide of type I collagen (CTx) from month 3 to month 12 (changes derived from a graph were approximately $70 \%$ and $105 \%$ respectively), and an early and persistent decrease in the alendronate group (58\% at month 1 , approximately $70 \%$ at month 12 ) and in the $\mathrm{PTH}+$ alendronate group (approximately 50\% throughout the trial). ${ }^{20}$ At the end of the second year of the PaTH study, all the groups showed similar values below baseline, except the PTH/placebo group, which returned to baseline values, and these were significantly higher than the other groups. ${ }^{21}$ Urinary NTx significantly increased also in PTH-treated patients in the POWER study (65\% at month 12 and 69\% at month 24).22

\section{Safety}

When a new pharmacological therapy is administered to large populations of patients it is mandatory to assess the safety of the treatment. For PTH (1-84) the principal concerns are with hypercalcemia and hypercalciuria, but also osteogenic sarcoma and hyperuricemia. ${ }^{34}$

The overall tolerability of PTH (1-84) in the trials was good. A similar number of patients experienced adverse events in the TOP study in the PTH and placebo groups (94.0\% vs 92.9\%), ${ }^{18}$ in the PaTH study in all treatment groups (percentage not indicated), ${ }^{21}$ and in the POWER study in both treatment groups $(96 \%$ PTH + HT vs 93\% HT all events, $26.7 \%$ vs $17.8 \%$ serious adverse events). ${ }^{22}$

The patients who discontinued the treatment in the TOP study because of adverse events comprised approximately $16 \%$ of the PTH group (nausea $4.4 \%$, headache $1.4 \%$, vomiting $1.1 \%$, dizziness $0.8 \%$, and elevated serum calcium $2.4 \%$ or urine calcium levels $0.5 \%$ ) and $12 \%$ of the placebo group (only $2 \%$ for these combined events). ${ }^{18}$ 


\section{Osteogenic sarcoma}

The pivotal registration trial for teriparatide was stopped due to the appearance of osteogenic sarcoma in all the Fischer strain of rats receiving lifelong high-dose teriparatide. ${ }^{35-37}$ Osteogenic sarcoma was also seen in the PTH 1-84 preclinical animal data in the rat, with a dose-responsive increased incidence (50 to $100 \mu \mathrm{g} /$ day). A noncarcinogenic dose of PTH (1-84) $10 \mu \mathrm{g} /$ day was identified. ${ }^{38}$

In humans only one case of osteogenic sarcoma has been reported in patients treated with teriparatide. The diagnosis was made in the biopsy of a lung lesion in a female smoker, but the bone source was not confirmed. ${ }^{39}$ Since there are about 800,000 patients worldwide on teriparatide, and the incidence of osteogenic sarcoma in the adult population is $1 / 250,000 /$ year, ${ }^{40}$ there is no evidence that this case was related to teriparatide. ${ }^{34}$

Furthermore, no abnormal histopathologic findings were reported in iliac crest biopsies after 12 and 18 months of PTH therapy in a subset of 40 women in the TOP trial ${ }^{18}$ and after 36 months in 14 patients in the TRES study. ${ }^{41}$

In 2008 a Finnish group reported a case of a malignant myeloma in a patient after treatment for osteoporosis with teriparatide. They were unable to demonstrate any direct association, though they found it intriguing that similar mechanisms (RANKL/RANK/OPG system) are involved in the development of myeloma and during teriparatide treatment. ${ }^{42}$

It seems that there is no real concern about PTH having a carcinogenic action in the dose and treatment duration approved in humans, but continued monitoring is advisable.

\section{Hypercalcemia}

The principal physiological role of PTH is to raise calcemia during fasting periods, so transitory hypercalcemia is expected to occur 4 to 6 hours after PTH injection. ${ }^{34}$ However, an unexpected persistent hypercalcemia was reported in a percentage of patients in all trials in which PTH was used to treat osteoporosis. ${ }^{34,43}$ In the TOP trial the number of patients with elevated serum calcium concentration $(>2.66 \mathrm{mmol} / \mathrm{L}$ or $10.7 \mathrm{mg} / \mathrm{dL}$ ) in the PTH (1-84) treated group was $27.8 \%$ compared with $4.5 \%$ in the placebo group. ${ }^{18}$ This incidence is greater than that reported in teriparatide trials and in the $\mathrm{PaTH}$ study $(12 \%),{ }^{20}$ and it has been attributed to different levels of calcemia at baseline. In the TOP study baseline calcemia was greater than 2.55 to $2.66 \mathrm{mmol} / \mathrm{L}$ in $8 \%$ of women, while $12 \%$ had hypercalciuria. ${ }^{16,44}$ The incidence of hypercalcemia in the PTH (1-84) trials is shown in Table 1.

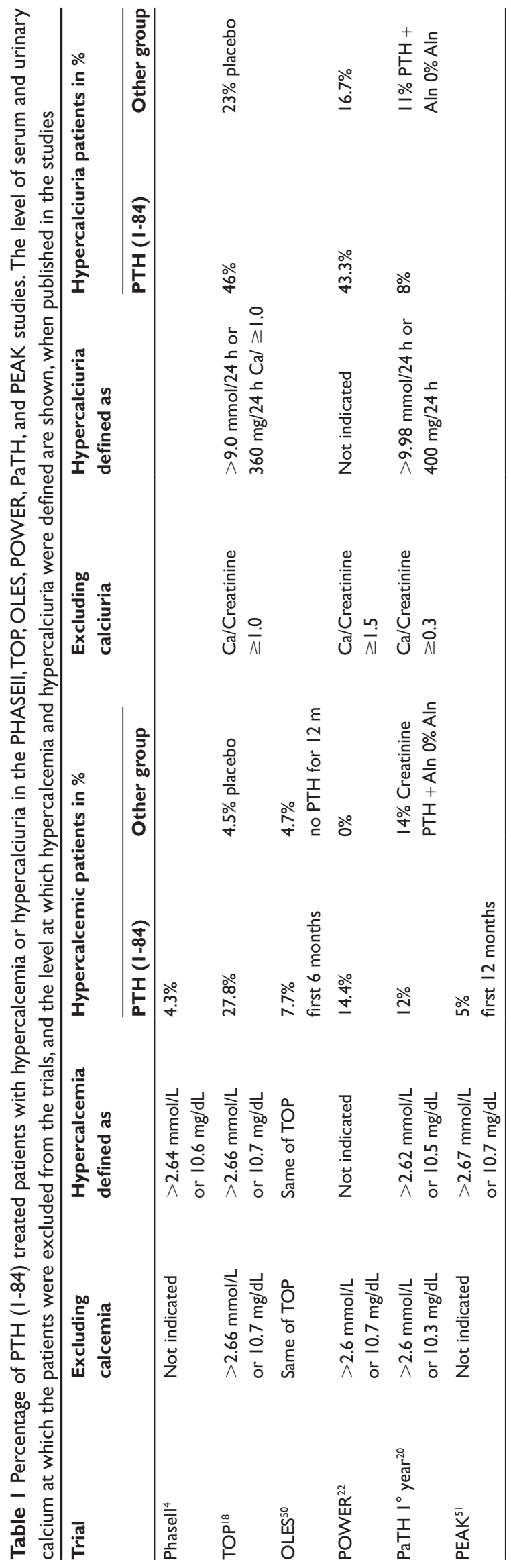


An analysis of the data from the PaTH trial focusing on hypercalcemia ${ }^{45}$ suggests that hypercalcemia is more frequent in women with higher baseline serum calcium or $1,25(\mathrm{OH})_{2} \mathrm{D}$ levels. The risk of hypercalcemia doubled with each $0.13 \mathrm{mmol} / \mathrm{L}$ (or $0.5 \mathrm{mg} / \mathrm{dL}$ ) increase in baseline serum calcium and with each increase in baseline serum $1,25(\mathrm{OH})_{2} \mathrm{D}$ concentration of $10 \mathrm{pg} / \mathrm{mL}$. The frequency of clinically relevant hypercalcemia, ie, $>11.2 \mathrm{mg} / \mathrm{dL}$, was similar in the PTH-alone and in the alendronate and PTH groups (4\% and 3.4\% respectively). This study also confirmed that changes in calcium levels follow a time pattern, rising at the first month, reaching a maximum at the third month, and decreasing to baseline values after 12 months.$^{45}$ Serum calcium level was found to be normal at a second measurement, without any intervention, in $58 \%$ of the previously hypercalcemic women and, after discontinuation of calcium and vitamin D supplementation, in $38 \%$. In 1 patient it was necessary to decrease the PTH injection frequency to every other day. Hypercalcemia recurred in 4 patients and only in 1 was it necessary to suspend calcium supplementation. ${ }^{45}$ In the PaTH study the protocol included a specific algorithm in case of elevated levels of serum and urinary calcium - the first step is to repeat the serum calcium measurement, and if hypercalcemia persists, the second step is to discontinue calcium and vitamin D, then to reduce PTH to every other day, and, finally, to discontinue $\mathrm{PTH} .{ }^{20,45}$

A 12-month, open label, head-to-head study, comparing the effect of PTH (1-84) and teriparatide in 70 women with severe osteoporotic vertebral fractures, recently reported a similar incidence (31\% and $29 \%$, respectively) of patients with hypercalcemia (ionized calcium $>1.3 \mathrm{mmol} / \mathrm{L}$ ). In the PTH (1-84)-treated group the mean concentration of ionized calcium was significantly higher throughout the study. ${ }^{46}$

Summarizing, hypercalcemia is frequently reported in PTH (1-84)-treated patients, but usually has no clinical consequences. The frequency of hypercalcemia is clearly related to the baseline level of serum calcium, so it is important to evaluate serum calcium before starting PTH (1-84) therapy. In the presence of high level of serum calcium $(>1.3$ to $10.5 \mathrm{mg} / \mathrm{dL}$ ) the risk of hypercalcemia during treatment is real and, without other equally valid therapeutic options, repeated measurements of serum calcium level are mandatory. In any case, all treated patients should have their serum calcium measured in the first month and, on the basis of the result further measurements should be planned. The blood for serum calcium should be drawn at least 16 hours after PTH injection. ${ }^{34}$ If hypercalcemia develops, the PaTH algorithm ${ }^{18}$ or the one proposed by Miller ${ }^{34}$ may be applied.

\section{Hypercalciuria}

In the TOP study hypercalciuria occurred in $46 \%$ and $23 \%$ of women receiving PTH and placebo, respectively. ${ }^{18} \mathrm{In}$ the POWER trial hypercalciuria was detected in $43.3 \%$ of the HT + PTH group and in $16.7 \%$ of the HT group. In the PaTH study, after year one, 9\% of enrolled women developed hypercalciuria, $8 \%$ in the PTH-alone group, $11 \%$ in the combined therapy group, and none in the alendronate group. ${ }^{20,45}$ Hypercalciuria resolved after discontinuing calcium and vitamin D in 12 patients (80\%), after decreasing PTH injection frequency in 2 patients, and did not recur when daily frequency was resumed, and without intervention in the remainder. Only 2 patients in the PTH-alone group had concurrent hypercalcemia and hypercalciuria. Mean urine calcium concentration significantly increased in both the PTH-alone and the PTH and alendronate groups, but there was no significant difference between the two groups. The risk of hypercalciuria increased by $50 \%$ with each $50 \mathrm{mg} /$ day increase in baseline urinary calcium (relative risk 1.5; $95 \%$ CI 1.1 to 2.0$).^{45}$

Data on hypercalciuria in the trials with PTH (1-84) are shown in Table 1.

\section{Hyperuricemia}

An increase in serum uric acid above the normal range was seen in the PTH group in the TOP study by month 18 , approximately $20 \%$ compared to $4.5 \%$ with placebo. ${ }^{18}$ In the PaTH study, too, a similarly ( $61 \mathrm{mmol} / \mathrm{L})$ significant increase in the mean serum uric acid concentration was seen in both the PTH + alendronate and the PTH-alone groups, and there were 2 patients with gout in the combination therapy group and 1 in the PTH group. ${ }^{20}$

\section{Other adverse events}

Other common adverse events in PTH (1-84) treated patients are nausea, headache, dizziness, back or limb pain, injection site complications, and fatigue.

In the phase II trial a non dose-related injection site reaction was the most common complaint in 134 of 217 women, but this event is not so widely reported in other studies. ${ }^{4}$

In the TOP study, PTH (1-84)-treated patients complained more frequently of nausea and vomiting than the placebo group patients $(22.6 \%$ vs $9.2 \% ; P<0.001$ and $8.0 \%$ vs $4.4 \%$, respectively). ${ }^{18}$ In the POWER study, nausea was more frequent in the HT + PTH group than in the HT-alone 
group (25.6\% vs 3.3\%). ${ }^{22}$ Headache frequency was higher in PTH-treated patients in the TOP study, $28.5 \%$ vs $23 \%$ in the placebo group $(P=0.001) .{ }^{18}$ No differences in headache frequency between various groups were reported in the PaTH study. ${ }^{20,21}$

In the TOP study PTH induced a slight reduction in hematocrit, hemoglobin, and erythrocyte count at all time points. No serious alterations in cardiac rhythm, repolarization, or intraventricular conduction were reported. ${ }^{18}$

\section{Adherence to treatment}

Adherence to treatment and compliance in the patients treated with PTH (1-84) in the clinical trials is high, when reported.

In the phase II trial compliance was very high, at $94.8 \%$ to $97.6 \%$ in all PTH groups, $14 \%$ of the patients discontinued the treatment, only $38 \%$ of these due to an adverse reaction and $48 \%$ due to study noncompliance. ${ }^{4}$

In the TOP study, 824 women out of $1286(64 \%)$ in the PTH-treated group completed the trial; 186 (14.5\%) discontinued drug therapy within 3 months. At the time of discontinuation the rate of patients treated with refracted dose regimens, adopted after adverse events, was higher in PTH groups $-21 \%$ and $5 \%$ of patients took injections every other day in the PTH and placebo group, respectively, and $13 \%$ and $4 \%$ when the injection was less frequent. Comparing the causes of discontinuation with the placebo group the number of drop-outs due to adverse events doubled, but serious adverse events, fractures, and confirmed bone loss were more frequent in the placebo group. ${ }^{18}$

In the POWER study the women who completed the study amounted to $62 \%$ in the PTH + HT group and $93 \%$ in the HT group, and overall compliance was high $(96 \%$ and $97 \%$ respectively). ${ }^{22}$

In the PaTH study $95 \%$ of patients completed the first year visit, and $75 \%$ fully adhered to treatment by injection. ${ }^{20}$

An improvement in patient compliance and therapy costs could be achieved by a refraction of injection frequency. A recent double-blind, randomized, placebo-controlled trial verified that less frequent PTH administration increased lumbar spine BMD, radial trabecular bone, and bone formation markers in 50 postmenopausal women aged 45 to 70 years with a femoral neck BMD T-score between -1.0 and -2.0 . The administration pattern comprised in one month of daily treatment with PTH (1-84) $100 \mu \mathrm{g}$, followed by weekly injections, same dose, for 11 months. ${ }^{47}$

A recent study using the choice-format conjoint (a surveybased stated-preference method that focuses on different features of the treatment from a patient's perspective) was conducted to measure postmenopausal osteoporosis patients' preferences. The preliminary data analysis of the responses from 102 German patients focusing on PTH-specific aspects indicates that postmenopausal osteoporotic patients' preferences are influenced mostly by features of PTH treatment such as 'storage requirements', 'reusability of the pen,' and 'dexterity/easiness for patients to use the pen'. ${ }^{48}$

These results confirm that an easy-to-use injection pen is important to improve patient adherence. The injection pen of commercially available PTH (1-84) uses a simple mechanism for daily administration, and refrigeration is not strictly necessary, but it must be refilled once a month. ${ }^{17}$

Large differences still exist in the number of patients receiving $\mathrm{PTH}$ across different countries, due to differing accessibility to PTH as a result of a combination of restrictive schedules of reimbursement, high cost, and physicians' lack of knowledge of the potential benefits of PTH. ${ }^{49}$

\section{Conclusions}

Full length PTH, available for the treatment of severe postmenopausal osteoporosis in Europe, is generally a well tolerated and effective treatment for postmenopausal osteoporosis. The number of clinical studies is still limited because it is not approved as a therapeutic agent in the US. ${ }^{16}$

The efficacy of PTH (1-84) in the prevention of vertebral fractures is well demonstrated, and similar to that of teriparatide. ${ }^{34}$ An effective role in prevention of femoral fractures has not yet been documented, but a decrease in nonvertebral fractures was seen in high-risk patients, ${ }^{40}$ and some data suggest that PTH (1-84) improves femoral strength. ${ }^{45}$ DXA scans have clearly demonstrated the effect on bone mass at the spine, and to a lesser extent at the hip. ${ }^{18,20,21}$ The increase in volumetric density and bone volume in trabecular sites is evident, but a bone volume increase has also been detected in cortical sites, which may explain hip fracture reduction with PTH treatment. ${ }^{39,16}$ BMD constantly increases with the continuation of the treatment until 36 months, ${ }^{18,20,22,23}$ but, after therapy discontinuation, the BMD decreases if an antiresorptive treatment is not started. ${ }^{21,23}$

Hypercalcemia and hypercalciuria are frequent consequences of PTH treatment, but rarely have clinical effects. They are usually well controlled by reducing calcium and vitamin D supplementation. Calcium levels require only periodic measurements. ${ }^{36}$ Other adverse events, such as nausea and vomiting, dizziness, headache, limb pain, and hyperuricemia, do not frequently interfere with the continuation of the treatment. 


\section{Disclosure}

The author discloses no conflicts of interest.

\section{References}

1. Silverberg SJ, Shane E, de la Cruz L, et al. Skeletal disease in primary hyperparathyroidism. J Bone Miner Res. 1989;4:283-291.

2. Dempster DW, Parisien M, Silverberg SJ, et al. On the mechanism of cancellous bone preservation in postmenopausal women with mild primary hyperparathyroidism. J Clin Endocrinol Metab. 1999;84:1562-1566.

3. Bilezikian JP, Silverberg SJ, Shane E, et al. Characterization and evaluation of asymptomatic primary hyperparathyroidism. J Bone Miner Res. 1991;6 Suppl 2:S85-S89; discussion S121-S124.

4. Hodsman AB, Hanley DA, Ettinger MP, et al. Efficacy and safety of human PTH-(1-84) in increasing bone mineral density in postmenopausal osteoporosis. J Clin Endocrinol Metab. 2003;88:5212-5220.

5. Dempster DW, Cosman F, Parisien M, et al. Anabolic actions of PTH on bone. Endocr Rev. 1993;14:690-709.

6. Tam CS, Heersche JN, Murray TM, Parsons JA. PTH stimulates the bone apposition rate independently of its resorptive action: differential effects of intermittent and continuous administration. Endocrinology. 1982;110:506-512.

7. Calvo MS, Eastell R, Offord KP, et al. Circadian variation in ionized calcium and intact PTH: evidence for sex differences in calcium homeostasis. J Clin Endocrinol Metab. 1991;72:69-76.

8. Hock JM, Gera I. Effects of continuous and intermittent administration and inhibition of resorption on the anabolic response of bone to PTH. J Bone Miner Res. 1992;7:65-72.

9. Potts JT. PTH: past and present. $J$ Endocrinol. 2005;187:311-325.

10. Stilgren LS, Rettmer E, Eriksen EF, Hegedüs L, Beck-Nielsen H, Abrahamsen B. Skeletal changes in osteoprotegerin and receptor activator of nuclear factor-kappaB ligand mRNA levels in primary hyperparathyroidism: effect of parathyroidectomy and association with bone metabolism. Bone. 2004;35:256-265.

11. Ma YL, Cain RL, Halladay DL, et al. Catabolic effects of continuous human PTH (1-38) in vivo is associated with sustained stimulation of RANKL and inhibition of osteoprotegerin and gene-associated bone formation. Endocrinology. 2001;142:4047-4054.

12. Onyia JE, Miles RR, Yang X, et al. In vivo demonstration that human PTH 1-38 inhibits the expression of osteoprotegerin in bone with the kinetics of an immediate early gene. $J$ Bone Miner Res. 2000;15:863-871.

13. Locklin RM, Khosla S, Riggs BL. Mechanisms of biphasic anabolic and catabolic effects of PTH (PTH) on bone cells. Bone. 2001;28(Suppl):S80.

14. Jilka RL, Weinstein RS, Bellido T, Roberson P, Parfitt AM, Manolagas SC. Increased bone formation by prevention of osteoblast apoptosis with PTH. J Clin Invest. 1999;104:439-446.

15. Buxton EC, Yao W, Lane NE. Changes in serum receptor activator of nuclear factor-kappaB ligand, osteoprotegerin, and interleukin-6 levels in patients with glucocorticoid-induced osteoporosis treated with human PTH (1-34). J Clin Endocrinol Metab. 2004;89:3332-3336.

16. Adami S. Full length PTH, PTH (1-84), for the treatment of severe osteoporosis in postmenopausal women. Curr Med Res Opin. 2008;24:3259-3274.

17. European Medical Agency - Product Information 27/11/2007 PreotactH-C-659-N-04. URL: http://www.emea.europa.eu/humandocs/PDFs/ EPAR/preotact/H-659-PI-en.pdf.

18. Greenspan SL, Bone HG, Ettinger MP, et al. Effect of recombinant human PTH (1-84) on vertebral fracture and bone mineral density in postmenopausal women with osteoporosis: a randomized trial. Ann Intern Med. 2007;146:326-339.

19. Roux C, Clausen J. Sustained effect of PTH (1-84) on the risk of vertebral fractures 12 months after cessation of therapy. Calcified Tissue Int. 2007;80(Suppl 1):S146.
20. Black DM, Greenspan SL, Ensrud KE, et al. The effects of PTH and alendronate alone or in combination in postmenopausal osteoporosis. N Engl J Med. 2003;349:1207-1215.

21. Black DM, Bilezikian JP, Ensrud KE, et al. One year of alendronate after one year of PTH (1-84) for osteoporosis. N Engl J Med. 2005;353: $555-565$.

22. Fogelman I, Fordham JN, Fraser WD, et al. PTH(1-84) Treatment of Postmenopausal Women with Low Bone Mass Receiving Hormone Replacement Therapy. Calcified Tissue Int. 2008;83:85-92.

23. Jodar-Gimeno E, Leib ES, Zanchetta JR, Mautalen CA, Greisen H. Continued increase in hip BMD after 36 Months of PTH(1-84) treatment in Postmenopausal women. Calcified Tissue Int. 2008;82(Suppl 1):S231.

24. Hosking D, Brandi M, Díaz-Curiel M, Felsenberg D, Hyldstrup LH. One year of treatment with $\mathrm{PTH}(1-84)$ and the change in lumbar spine BMD in postmenopausal women with primary osteoporosis after pretreatment with a bisphosphonate. Bone. 2009;44(Suppl 2):S428.

25. Minisola S, Bone HG, Zanchetta JR, et al. Influence of lumbar spine BMD at baseline on the incidence of new vertebral fractures after 18 months treatment with $\mathrm{PTH}(1-84)$. Results from the top study. Calcified Tissue Int. 2008;82(Suppl 1):S238.

26. Neer RM, Arnaud CD, Zanchetta JR, et al. Effect of PTH (1-34) on fractures and bone mineral density in postmenopausal women with osteoporosis. N Engl J Med. 2001;344:1434-1441.

27. Roux C, Illera-martin O, Clausen J. Efficacy of PTH (1-84) on vertebral and non-vertebral fractures in postmenopausal women with a high risk of vertebral fractures. Calcified Tissue Int. 2007;80(Suppl 1):S48.

28. Moen MD, Scott LJ. Recombinant full-length PTH (1-84). Drugs. 2006;66(18):2371-2381.

29. Black D, Bilezikian J, Greenspan S, et al. Gains in lumbar spine BMD in highly compliant patients treated with PTH (1-84). Bone. 2009;44(Suppl 2):S428-S429.

30. Khoo BC, Beck TJ, Qiao QH, et al. In vivo short-term precision of hip structure analysis variables in comparison with bone mineral density using paired dual-energy X-ray absorptiometry scans from multicenter clinical trials. Bone. 2005;37:112-121.

31. van Londen GJ, Palermo L, Beck TJ, et al. Hip structural analysis and volumetric qct in postmenopausal women from the PaTH study: monoor combination therapy with alendronate and PTH. J Bone Miner Res. 2008; ASBMR Annual Meeting Abstracts: M306.

32. Keaveny TM, Hoffmann PF, Singh M, et al. Femoral bone strength and its relation to cortical and trabecular changes after treatment with PTH, Alendronate, and their combination as assessed by Finite Element Analysis of Quantitative CT Scans. J Bone Miner Res. 2008;23:1974-1982.

33. Bilezikian JP. Combination anabolic and antiresorptive therapy for osteoporosis: opening the anabolic window. Curr Osteoporos Rep. 2008;6:24-30.

34. Miller PD. Safety of PTH for the treatment of osteoporosis. Curr Osteoporos Rep. 2008;6:12-16.

35. Vahle JL, Sato M, Long GG, et al. Skeletal changes in rats given daily subcutaneous injections of recombinant human PTH (1-34) for 2 years and relevance to human safety. Toxicol Pathol. 2002;30:312-321.

36. Vahle JL, Long GG, Ma YL, Sato M. Bone neoplasms in F344 rats given teriparatide [rhPTH (1-34)] are dependent on duration of treatment and dose. Toxicol Pathol. 2004;32:426-438.

37. Neer RM, Arnaud CD, Zanchetta JR, et al. Effect of PTH (1-34) on fractures and bone mineral density in postmenopausal women with osteoporosis. N Engl J Med. 2001;344:1434-1441.

38. Wilker CE, Jolette J, Smith SY, et al. No observable carcinogenic effect dose level identified in Fischer 344 rats following daily treatment with PTH (1-84) for 2 years: role of the c-terminal PTH receptor? J Bone Miner Res. 2004;19(Suppl 1):S98.

39. Harper K, Krege JH, Marcus R, Mitlak BH. Osteosarcoma and teriparatide? J Bone Miner Res. 2007;22:334.

40. Damron TA, Ward WG, Stewart A. Osteosarcoma, chondrosarcoma, and Ewing's sarcoma: National Cancer Data Base Report. Clin Orthop Relat Res. 2007;459:40-47. 
41. Recker RR, Zanchetta CA, Mautalen CA, et al. Safety indices from iliac crest biopsies from postmenopausal osteoporotic women after 36 months of treatment with PTH(1-84). Bone. 2009;44(Suppl 2): S431-S432.

42. Forslund T, Koski AM, Koistinen A, Sikiö A. Malignant myeloma in a patient after treatment for osteoporosis with teriparatide; a rare coincidence. Clinical Medicine: Case Reports. 2008;1:119-122.

43. Vestergaard P, Jorgensen NR, Mosekilde L, Schwarz P. Effects of PTH alone or in combination with antiresorptive therapy on bone mineral density and fracture risk - a meta-analysis. Osteoporos Int. 2007;18: 45-57.

44. Verhaar HJJ, Lems WF. PTH-analogs: comparable or different? Arch Gerontol Geriatr. 2009;49(2):e130-e132.

45. Antoniucci DM, Sellmeyer DE, Bilezikian JP, et al. Elevations in Serum and Urinary Calcium with PTH (1-84) with and without Alendronate for Osteoporosis. J Clin Endocrinol Metab. 2007;92(3):942-947.

46. Bevilacqua M, Dominguez LJ, Chebat E, et al. Treatment with daily subcutaneous teriparatide (1-34) compared with daily subcutaneous PTH (1-84) in women with severe postmenopausal osteoporosis: a randomized, head-to-head study. Osteoporos Int. 2009;20(Suppl 1):S173.
47. Black DM, Bouxsein ML, Palermo L, et al. Randomized trial of once-weekly PTH (1-84) on bone mineral density and remodeling. J Clin Endocrinol Metab. 2008;93:2166-2172.

48. Asmussen M, Benhamou CL, Törring O, Minisola S, Hyldstrup LH. Preferences from postmenopausal osteoporosis patients for administration of PTH treatment. Calcif Tissue Int. 2008;82(S1):P400.

49. Hosking D, Alonso CG, Brandi ML. Management of osteoporosis with PTH: treatment and prescription patterns in Europe. Curr Med Res Opin. 2009;25(1):263-270.

50. Perez-Edo L, Zanchetta JR, Mautalen CA, Bolognese MA, Greisen H Safety of PTH (1-84) after 24 months therapy and a 12 months follow-up period. Calcif Tissue Int. 2008;82(Suppl 1):S241-S242.

51. Díaz-Curiel M, Hosking D, Brandi M, Hyldstrup LH, Felsenberg D. Serum calcium levels in postmenopausal women with osteoporosis after 1, 6 and 12 months of PTH (1-84) treatment - the latest results from the PEAK trial. Bone. 2009;44(Suppl 2):S435-S436.
International Journal of Women's Health

\section{Publish your work in this journal}

The International Journal of Women's Health is an international, peerreviewed open-access journal publishing original research, reports, reviews and commentaries on all aspects of women's healthcare including gynaecology, obstetrics, and breast cancer. Subject areas include: Chronic conditions (migraine headaches, arthritis, osteoporosis);

\section{Dovepress}

Endocrine and autoimmune syndromes; Sexual and reproductive health; Psychological and psychosocial conditions. The manuscript management system is completely online and includes a very quick and fair peer-review system. Visit http://www.dovepress.com/ testimonials.php to read real quotes from published authors. 